\section{Bonne Année!}

Le ministre de l'Environnement David Anderson a rendu public en décembre, un document pour fins de discussion "Le Plan canadien pour la protection des espèces en péril." On peut obtenir de l'information sur le site Internet d'Environnement Canada à www.ec.gc.ca ou en téléphonant au 1-800-6686767. Le sommaire exécutif indique "que la stratégie proposée établit une approche durable à long terme que ferait des mesures incitatives, d'intendance et volontaires l'option préférée pour protéger les espèces en péril ou en voie d'extinction ainsi que leurs habitats essentiels. La loi procurait un cadre de travail pour protéger les espèces en péril ainsi que des mesures de protection selon le besoin."

La stratégie contient trois parties pour protéger les espèces: 1) s'appuyer sur des partenariats avec les provinces et les territoires grâce à l'Accord de protection des espèces en péril; 2) faire la promotion de programmes d'intendance et d'incitation destinées à aider les propriétaires terriens, les particuliers, les peuples autochtones et les organismes participant à la protection des espèces et de leurs habitats; et 3 ) présenter un nouveau projet de loi sur les espèces en péril.

Le ministre a proposé que la loi couvre les points suivants:

a) une liste des espèces en péril telle qu'établies par le COSEWIC, cependant le gouvernement fédéral pourrait adopter une liste différente selon certaines justifications,

b) les interdits s'appliqueraient à toutes les espèces en péril ou en voie d'extinction de la liste dans les domaines de juridiction fédérale ou sur les terres fédérales, cependant un filet de sécurité protégerait les espèces en péril ou en voie d'extinction qui ne sont pas protégées dans le faits par les lois ou les règlements provinciaux ou territoriaux,

c) la planification de la récupération pourrait faire partie du processus,

d) le filet de sécurité des habitats essentiels s'appliquerait lorsque le gouvernement utiliserait une loi, un règlement ou d'autres mesures pour protéger un habitat sur des terres fédérales ou sur terres publiques provinciales, l'intendance serait toujours le principal moyen de protection des habitats sur terres privées, toutefois en dernier recours lorsque ces mesures ne fonctionnent pas, le gouvernement du Canada aurait à sa disposition des mesures permettant d'interdire la destruction d'un habitat essentiel,

e) un dédommagement serait offert lorsque requis, cependant les compensations diffèrent des initiatives d'intendance et ne devraient pas être confondues,

f) La loi comprendrait des mesures pour s'assurer de la conformité avec cette dernière et l'application effective de ses provisions tout en gardant en tête que le gouvernement s'est également impliqué à travailler en collaboration pour éviter et résoudre les disputes qui pourraient surgir lors de l'application de la loi.

Il ne s'agit que des "faits saillants" des faits saillants, et je voudrais encourager les membres à se procurer une copie du document de discussions. Le ministre Anderson prévoit déposer la loi en février 2000. L'Institut est à finaliser un mémoire sur le sujet qui sera distribué aux membres lorsqu'il aura reçu l'approbation du Conseil national d'administration.
Le ministre Anderson a connu un mois de décembre chargé, puisqu'il a également dévoilé une révision de la loi canadienne sur l'évaluation environnementale le 14 décembre 1999. Le communiqué de presse indique que la révision comprendra divers éléments, dont un site Internet interactif, permettant d'obtenir efficacement de l'information et l'opinion de tous les citoyens des zones urbaines et rurales du Canada, des rencontres de consultation publique nationale qui auront lieu dans 19 agglomérations au canada. Un document pour fins de discussion sur la consultation publique est disponible auprès de l'Agence canadienne d'évaluation environnementale (tél.) 819-953-0179 (appel à frais virés) ou sur leur site Internet http://www.ceas.gc.ca

La loi canadienne sur l'évaluation environnementale est entrée en vigueur en janvier 1995 et nécessite une révision qui devrait débuter au plus tard en janvier 2000. "La loi et ses règlements s'appliquent à tous les projets où le gouvernement fédéral joue un rôle de décideur, que ce soit à titre de promoteur, de gestionnaire du terrain, de bailleur de fonds ou de législateur. Depuis la mise en application de la loi, plus de 25000 évaluations environnementales ont été réalisées."

Les consultations débuteront le 31 janvier pour se terminer le 15 mars 2000.

Avant de terminer, la date limite pour les nominations des prix nationaux de l'Institut est le ler mai 2000. L'information sur les critères et les procédures de mise en nomination peut être retrouvée sur le site Internet de l'Institut http://www.cififc.org, ou il est possible d'obtenir une brochure auprès des présidents ou directeurs de section, ou au bureau national. Les prix nationaux de l'Institut dans les catégories Contribution canadienne, Contribution scientifique, Contribution internationale, James M. Kitz et Contribution de groupe sont remis à des individus ou à des groupes d'individus qui ont apporté une contribution remarquable ou réalisé un projet important en foresterie. Nous désirons recevoir les nominations au bureau national d'ici le ler mai 2000. Les Prix seront remis lors du banquet de l'Institut à Corner Brook, Terre-Neuve en août 2000.

\section{Come visit CIF/IFC at http://www.cif-ifc.org}

read about CIF/IFC activities \& Section

News

a find Working Group contacts

$\square$ read CIF/IFC Position Papers

- learn about Continuing Education Opportunities

\ explore Job Opportunities

become a member

view links to other sites

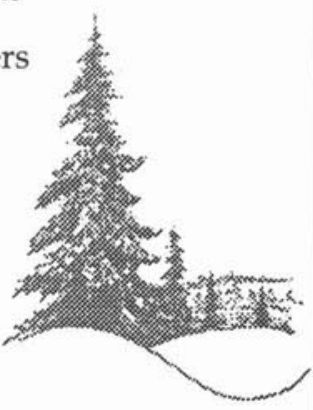

